\title{
ON DERIVATIONS AND COMMUTATIVITY IN PRIME RINGS
}

\author{
VINCENZO DE FILIPPIS
}

Received 29 March 2004

Let $R$ be a prime ring of characteristic different from 2, $d$ a nonzero derivation of $R$, and $I$ a nonzero right ideal of $R$ such that $[[d(x), x],[d(y), y]]=0$, for all $x, y \in I$. We prove that if $[I, I] I \neq 0$, then $d(I) I=0$.

2000 Mathematics Subject Classification: 16N60, 16W25.

1. Introduction. Let $R$ be a prime ring and $d$ a nonzero derivation of $R$. Define $[x, y]_{1}$ $=[x, y]=x y-y x$, then an Engel condition is a polynomial $[x, y]_{k}=\left[[x, y]_{k-1}, y\right]$ in noncommuting variables. A commutative ring satisfies any such polynomial, and a nilpotent ring satisfies one if $k$ is sufficiently large.

In this paper, we fix our attention on the Engel condition $\left[\left[d\left(x_{1}\right), x_{1}\right], x_{2}\right]$.

A well-known result of Posner [12] states that if $\left[\left[d\left(x_{1}\right), x_{1}\right], x_{2}\right]=0$, for all $x_{1}, x_{2} \in$ $R$, then $R$ is commutative. This result has led to many others which combine derivations with Engel-type conditions. In [14], Vukman showed that $R$ is commutative if $\operatorname{char}(R) \neq$ 2 and $\left[\left[d\left(x_{1}\right), x_{1}\right], x_{1}\right]=0$, for all $x_{1} \in R$. On the other hand, Lanski proved in [8] that if $\left[\left[d\left(x_{1}\right), x_{1}\right], x_{2}\right]=0$, for all $x_{1}$ in a noncommutative Lie ideal and $x_{2} \in R$, then either $R$ is commutative or $\operatorname{char}(R) \neq 2$ and $R$ satisfies the standard identity of degree 4 .

Several authors have studied what happens if the Engel condition is satisfied by the elements of a nonzero one-sided ideal of $R$. To be more specific, in [2] Bell and Martindale proved that if $R$ is semiprime and $\left[\left[d\left(x_{1}\right), x_{1}\right], x_{2}\right]=0$, for all $x_{1}$ in a nonzero left ideal and $x_{2} \in R$, then $R$ contains a nonzero central ideal. Later, Bell and Deng showed that the same conclusion holds if $R$ is semiprime with suitably restricted additive torsion and $\left[\left[d\left(x_{1}\right), x_{1}\right], x_{1}\right]$ falls in the center of $R$, for all $x_{1}$ in a nonzero left ideal of $R[4]$.

Clearly, the last two results state that if $R$ is prime then it is commutative.

The question of whether a ring is commutative or nilpotent, if it satisfies an Engel condition, goes back to the well-known work of Engel on Lie algebras [6, Chapter 2].

Here, we will examine what happens in case $[[d(x), x],[d(y), y]]=0$, for any $x, y \in$ $I$, a nonzero right ideal of $R$.

One cannot expect the conclusion that $R$ is commutative, as the following example shows.

EXAMPLE 1.1. Consider $R=M_{2}(F)$, the ring of all $2 \times 2$ matrices over the field $F$. Let $e_{i j}$ be the usual matrix unit in $R$ and $I=e_{11} R$. Any derivation $\delta: F \rightarrow F$ induces another one in $R=M_{2}(F)$ as follows: $d: M_{2}(F) \rightarrow M_{2}(F)$ such that $d\left(\sum_{i, j} r_{i j} e_{i j}\right)=\sum_{i, j} \delta\left(r_{i j}\right) e_{i j}$, 
for any matrix $A=\sum_{i, j} r_{i j} e_{i j}$, where $r_{i, j} \in F$. In this case,

$$
\left[\left[d\left(e_{11} x\right), e_{11} x\right],\left[d\left(e_{11} y\right), e_{11} y\right]\right]=0
$$

for any $x, y \in R$, but clearly $R$ is not commutative.

We will proceed by first proving the following theorem.

THEOREM 1.2. Let $R$ be a prime ring of characteristic different from $2, d$ a nonzero derivation of $R$, such that $[[d(x), x],[d(y), y]]=0$, for all $x, y \in R$. Then, $R$ is commutative.

Finally, in the second part of the paper, we will extend the previous theorem to a nonzero right ideal of $R$.

We will prove the following theorem.

THEOREM 1.3. Let $R$ be a prime ring of characteristic different from $2, d$ a nonzero derivation of $R$ and $I$ a nonzero right ideal of $R$ such that $[[d(x), x],[d(y), y]]=0$, for all $x, y \in I$. If $[I, I] I \neq 0$, then $d(I) I=0$.

The assumption $[I, I] I \neq 0$ is essential to the main result. In fact, consider Example 1.1 and notice that $\left[x_{1}, x_{2}\right] x_{3}$ is an identity for $I=e_{11} R$, but clearly $d(I) I=d\left(e_{11} R\right) e_{11}$ $R \neq 0$.

We first fix the following facts.

FACT 1. In what follows, we denote by $Q$ the Martindale quotients ring of $R$ and by $C=Z(Q)$ the extended centroid of $R$ (see [1, Chapter 2]). When $R$ is prime, all that we need here about these objects is that $R \subseteq Q, Q$ is prime, and $C$ is a field.

Let $T=Q *_{C} C\{X\}$ be the free product over $C$ of the $C$-algebra, $Q$, and the free $C$-algebra, $C\{X\}$, with $X$ a countable set consisting of noncommuting indeterminates $\left\{x_{1}, \ldots, x_{n}, \ldots\right\}$. The elements of $T$ are called generalized polynomial with coefficients in $Q . I, I R$, and $I Q$ satisfy the same generalized polynomial identities with coefficients in $Q$. For more details about these objects we refer the reader to [1, 3].

FACT 2. Any derivation of $R$ can be uniquely extended to a derivation of $Q$, and so any derivation of $R$ can be defined on the whole of $Q$ [1, Proposition 2.5.1]. Moreover, $Q$ is a prime ring as well as $R$ and the extended centroid $C$ of $R$ coincides with the center of $Q$ [1, Proposition 2.1.7, Remark 2.3.1].

FACT 3 (see Kharchenko [7]). Let $f\left(x_{1}, \ldots, x_{n}, d\left(x_{1}\right), \ldots, d\left(x_{n}\right)\right)$ be a differential identity of $R$. One of the following holds:

(1) either $d$ is an inner derivation in $Q$, in the sense that there exists $q \in Q$ such that $d(x)=[q, x]$, for all $x \in Q$ and $Q$ satisfies the generalized polynomial identity $f\left(x_{1}, \ldots, x_{n},\left[q, x_{1}\right], \ldots,\left[q, x_{n}\right]\right) ;$ or

(2) $R$ satisfies the generalized polynomial identity $f\left(x_{1}, \ldots, x_{n}, y_{1}, \ldots, y_{n}\right)$.

FACT 4 (see Lee [10]). I, IR, and IQ satisfy the same differential identities with coefficients in $Q$. 
In all that follows, unless stated otherwise, $R$ will be a prime ring of characteristic $\neq 2$, $d \neq 0$ a derivation of $R$ and $I$ a nonzero right ideal of $R$ such that $[[d(x), x],[d(y), y]]$ $=0$, for all $x, y \in I$.

2. The case $I=R$. In this section, we consider the case when $[[d(x), x],[d(y), y]]=$ 0 , for all $x, y \in R$ and prove Theorem 1.2.

Proof of TheOrem 1.2. Denote the differential polynomial

$$
\left[\left[d\left(x_{1}\right), x_{1}\right],\left[d\left(x_{2}\right), x_{2}\right]\right]=g\left(x_{1}, x_{2}, d\left(x_{1}\right), d\left(x_{2}\right)\right) .
$$

Then, $g\left(x_{1}, x_{2}, d\left(x_{1}\right), d\left(x_{2}\right)\right)$ is a differential identity on $R$.

Using Fact 3, one of the following holds:

(1) $d$ is an inner derivation in $Q$, induced by $c \in Q$ and $R$ satisfies the generalized polynomial identity

$$
g\left(x_{1}, x_{2},\left[c, x_{1}\right],\left[c, x_{2}\right]\right)
$$

(2) $R$ satisfies the generalized polynomial identity $g\left(x_{1}, x_{2}, y_{1}, y_{2}\right)$.

In this last case, $R$ satisfies the identity [[$\left.\left.y_{1}, x_{1}\right],\left[y_{2}, x_{2}\right]\right]$, that is, for any $r_{1}, r_{2}, r_{3}$, $r_{4} \in R,\left[\left[r_{1}, r_{2}\right],\left[r_{3}, r_{4}\right]\right]=0$.

Since $R$ is a polynomial identity (P.I.) ring, there exists a field $F$ such that $R$ and $M_{t}(F)$, the ring of $t \times t$ matrices over $F$, satisfy the same polynomial identities.

Suppose $t \geq 2$ and choose $r_{1}=e_{11}, r_{2}=e_{12}, r_{3}=e_{21}, r_{4}=e_{11}$. Then, we obtain the following contradiction:

$$
0=\left[\left[e_{11}, e_{12}\right],\left[e_{21}, e_{11}\right]\right]=e_{11}-e_{22} \neq 0 .
$$

Therefore, we must have $t=1$ and so $R$ is commutative.

Now, let $d$ be the inner derivation induced by an element $c \in Q$. Thus,

$$
\left[\left[c, r_{1}\right]_{2},\left[c, r_{2}\right]_{2}\right]=0
$$

for any $r_{1}, r_{2} \in R$, that is, $R$ satisfies a nontrivial generalized polynomial identity. By [11], it follows that $S=R C$ is a primitive ring with $\operatorname{soc}(R)=H \neq 0$ and $e H e$ is a simple central algebra finite-dimensional over $C$, for any minimal idempotent element $e \in S$. Moreover, we may assume $H$ noncommutative, otherwise also $R$ must be commutative. Notice that $H$ satisfies $\left[\left[c, x_{1}\right]_{2},\left[c, x_{2}\right]_{2}\right]$ (see, e.g., [9, proof of Theorem 1]).

Since $H$ is a simple ring, one of the following holds: either $H$ does not contain any nontrivial idempotent element or $H$ is generated by its idempotents.

In this last case, suppose that $H$ contains two minimal orthogonal idempotent elements $e, f$ so that $e H, f H$ are isomorphic $H$-modules. For all $x \in H$,

$$
0=\left[[c, e]_{2},[c, f x e]_{2}\right]=[c e+e c-2 e c e,-2 f x e c f x e] .
$$

Left multiplying by $e$, we have $-2 e c f x e c f x e=0$. This implies in particular $(e c f x)^{3}=$ 0 . From this, by [5], ec $f H=0$. By the primeness of $H$, this implies that, for any orthogonal idempotent elements of rank $1, e$ and $f$, ec $f=0$. Hence, $[c, e]=0$, for any 
idempotent $e$ of rank 1 , and $[c, H]=0$, since $H$ is generated by these idempotent elements. This argument gives the contradiction that $c \in C$ and $d=0$.

Therefore, $H$ cannot contain two minimal orthogonal idempotent elements and so $H=D$, for a suitable division ring $D$ finite dimensional over its center. This implies that $Q=H$ and $c \in H$. By [13, Theorem 2.3.29, page 131] (see also [9, Lemma 2]), there exists a field $F$ such that $H \subseteq M_{n}(F)$ and $M_{n}(F)$ satisfies $\left[\left[c, x_{1}\right]_{2},\left[c, x_{2}\right]_{2}\right]$, for $F$ a field. As we have just seen, if $n \geq 2$, then $c \in C$ and $d=0$. If $n=1$, then $H \subseteq F$ and we are also done.

On the other hand, if $H$ does not contain any nontrivial idempotent element, then $H$ is a finite-dimensional division algebra over $C$ and $c \in H=R C=Q$. If $C$ is finite, then $H$ is a finite division ring, that is, $H$ is a commutative field and so $R$ is commutative too.

If $C$ is infinite, then $H \otimes_{C} F \cong M_{r}(F)$, where $F$ is a splitting field of $H$. In this case, a Vandermonde determinant argument shows that in $M_{r}(F)\left[\left[c, x_{1}\right]_{2},\left[c, x_{2}\right]_{2}\right]$ is still an identity. As above, one can see that if $r \geq 2$, then $c$ commutes with any idempotent element in $M_{r}(F)$. In this case, we have the contradiction $d=0$. In the other one, $H$ is commutative, as well as $R$.

3. The case $I$ is a right ideal of $R$. In this final section, we will prove the main theorem of the paper (Theorem 1.3).

For the rest of the paper, we now assume the conclusion of Theorem 1.3 to be false; our goal is to ultimately arrive at a contradiction. Thus, we will assume henceforth that $d(I) I \neq 0$. We begin with the following lemma.

LEMMA 3.1. $R$ is a ring satisfying a nontrivial generalized polynomial identity (GPI).

Proof. Suppose by contradiction that $R$ does not satisfy any nontrivial generalized polynomial identity. We divide the proof into two cases.

CASE 1. Suppose that $d$ is an inner derivation induced by an element $c \in Q$.

By the last assumption $d(I) I \neq 0$, there exists an element $b \in I$ such that $c b \neq 0$. Thus, $R$ satisfies the polynomial identity, $\left[[c, b x]_{2},[c, b y]_{2}\right]$. Moreover, we may assume $b \notin C$, otherwise $R$ should satisfy $\left[[c, x]_{2},[c, y]_{2}\right]$ which is a nontrivial generalized polynomial identity.

Expanding the previous GPI we get

$$
\begin{aligned}
& {\left[[c, b x]_{2},[c, b y]_{2}\right]} \\
& =\left(c(b x)^{2}+(b x)^{2} c-2 b x c b x\right)\left(c(b y)^{2}+(b y)^{2} c-2 b y c b y\right) \\
& \quad+\left(-c(b y)^{2}-(b y)^{2} c+2 b y c b y\right)\left(c(b x)^{2}+(b x)^{2} c-2 b x c b x\right) .
\end{aligned}
$$

Suppose that $\{b, c b\}$ are linearly $C$-dependent, then there exists $0 \neq \alpha \in C$ such that $c b=\alpha b$. In this case, $R$ satisfies

$$
(-\alpha b x b x+b x b x c)(-\alpha b y b y+b y b y c)+(\alpha b y b y+b y b y c)(-\alpha b x b x+b x b x c) .
$$


Since $b, c \notin C$, this last formula is a nontrivial generalized polynomial identity for $R$ (see [3]), a contradiction.

On the other hand, if $\{b, c b\}$ are linearly $C$-independent it follows, again by Chuang's results in $[3]$, that $\left[[c, b x]_{2},[c, b y]_{2}\right]$ is a nontrivial generalized polynomial identity for $R$. In any case, we have a contradiction.

CASE 2. Suppose now that $d$ is an outer derivation.

First, notice that if for all $t \in I$ there exists $\alpha_{t} \in C$ such that $d(t)=\alpha_{t} t$, then $[d(x), x]$ is an identity for $I$. This implies the contradiction that $R$ is commutative (as a consequence of [4]).

So, let $b \in I$ such that $\{b, d(b)\}$ are linearly $C$-independent.

By our assumption, we have that $[[d(b x), b x],[d(b y), b y]]=0$, and so

$$
0=[[d(b) x+b d(x), b x],[d(b) y+b d(y), b y]]
$$

By Fact 3 it follows that

$$
0=\left[\left[d(b) r_{1}+b r_{2}, b r_{1}\right],\left[d(b) r_{3}+b r_{4}, b r_{3}\right]\right]
$$

for all $r_{1}, r_{2}, r_{3}, r_{4} \in R$. In particular, $R$ satisfies the blended component

$$
[[d(b) x, b x],[d(b) y, b y]]
$$

which is a nontrivial generalized polynomial identity for $R$, because $\{b, d(b)\}$ are linearly $C$-independent, a contradiction.

Proposition 3.2. Without loss of generality, $R$ is simple and equal to its own socle, $I R=I$.

Proof. By Lemma 3.1, $R$ is GPI and so $Q$ has nonzero socle $H$ with nonzero right ideal $J=I H$ [11]. Note that $H$ is simple, $J=J H$, and $J$ satisfies the same basic conditions as $I$, in view of Fact 4. Now, just replace $R$ by $H, I$ by $J$, and we are done.

Now, we are ready to prove the main result.

Proof of Theorem 1.3. Since $I$ does not satisfy $\left[x_{1}, x_{2}\right] x_{3}$, there exist $a_{1}, a_{2}$, $a_{3} \in I$, such that $\left[a_{1}, a_{2}\right] a_{3} \neq 0$. Here, we suppose that $d(I) I \neq 0$, that is, there exist $a_{4}, a_{5} \in I$ such that $d\left(a_{4}\right) a_{5} \neq 0$ and we proceed to derive a contradiction. In view of Fact 3, we divide the proof into two cases.

CASE 1. If $d$ is an inner derivation induced by the element $q \in Q$, then $I$ satisfies the identity $\left[[q, x]_{2},[q, y]_{2}\right]$, moreover, $q I \neq 0$, since $d(I) I \neq 0$. Let $e^{2}=e \in I$. Thus, for all $y \in R,\left[[q, e]_{2},[q, e y(1-e)]_{2}\right]=0$, and left multiplying by $(1-e)$, we get $-2(1-$ $e) q e y(1-e) q e y(1-e)=0$. Since $\operatorname{char}(R) \neq 2$, it follows that $((1-e) q e y)^{3}=0$. By [5], $(1-e) q e R=0$ and by the primeness of $R,(1-e) q e=0$.

Let $r \in I$ and suppose $a r \neq 0$. Write $r R=e R, e^{2}=e \in I$, noting that $r=e r$. Then, $(1-e) q e=0$ implies $q r=e q r+(1-e) q r=e q r+(1-e) q e r=e q r$. Thus, we get $q I \subseteq I$. Let $\bar{I}=I / I \cap l_{R}(I) ; \bar{I}$ is a prime $C$-algebra with a derivation $\bar{d}$ such that $\bar{d}(\bar{x})=\overline{d(x)}$, for 
all $x \in I$. Therefore, we have $0=[\overline{[d(x), x]}, \overline{[d(y), y]}]$, for all $\bar{x}, \bar{y} \in \bar{I}$. By Theorem 1.2 , either $\bar{d}=0$ modulo $l_{R}(I)$, or $\bar{I}$ is commutative modulo $l_{R}(I)$. In the first case, we have $d(I) I=0$ and in the second one $[I, I] I=0$. In any case, we have a contradiction.

CASE 2. Now, we assume that the derivation $d$ is not inner.

By the regularity of $R$, there exists an element $e^{2}=e \in I R$ such that $e R=a_{1} R+a_{2} R+$ $a_{3} R+a_{4} R+a_{5} R$ and $e a_{i}=a_{i}$, for $i=1,2,3,4,5$.

By our assumption, we have that, for all $b \in I[[d(b x), b x],[d(b y), b y]]=0$. As we have seen in Lemma 3.1, in this case $R$ satisfies the blended component

$$
[[d(b) x, b x],[d(b) y, b y]]
$$

Therefore, for all $r_{1}, r_{2} \in R\left[\left[d(e) r_{1}, e r_{1}\right],\left[d(e) r_{2}, e r_{2}\right]\right]=0$ and left multiplying by $(1-e)$,

$$
(1-e) d(e) e r_{1} e r_{1} e r_{2}(1-e) d(e) e r_{2}(1-e)=0
$$

For $r_{1}=e$, we get $(1-e) d(e) e r_{2}(1-e) d(e) e r_{2}(1-e)=0$, which implies $((1-e) d(e) e R)^{3}$ $=0$. Again, by [5], $0=(1-e) d(e) e=(1-e) d(e)$. This implies $d(e) \in e R$ and $d(e R) \subseteq e R$.

Let $\varrho=e R, \bar{\varrho}=\varrho / \varrho \cap l_{R}(\varrho)$ with $l_{R}(\varrho)$ the left annihilator of $\varrho$ in $R$. Therefore, $\bar{\varrho}$ satisfies the differential identity $\overline{[[d(x), x],[d(y), y]]}$.

By Theorem 1.2, we have that either $\bar{d}=\overline{0}$ or $\bar{\varrho}$ is commutative. Therefore, we have that either $d(e R) e R=0$ or $[e R, e R] e R=0$.

On the other hand, we have that $\left[e a_{1}, e a_{2}\right] e a_{3}=\left[a_{1}, a_{2}\right] a_{3} \neq 0$ and also $d\left(e a_{4}\right) e a_{5}=$ $d\left(a_{4}\right) a_{5} \neq 0$. This contradiction completes the proof of the theorem.

\section{REFERENCES}

[1] K. I. Beidar, W. S. Martindale, III, and A. V. Mikhalev, Rings with Generalized Identities, Monographs and Textbooks in Pure and Applied Mathematics, vol. 196, Marcel Dekker, New York, 1996.

[2] H. E. Bell and W. S. Martindale, III, Centralizing mappings of semiprime rings, Canad. Math. Bull. 30 (1987), no. 1, 92-101.

[3] C.-L. Chuang, GPIs having coefficients in Utumi quotient rings, Proc. Amer. Math. Soc. 103 (1988), no. 3, 723-728.

[4] Q. Deng and H. E. Bell, On derivations and commutativity in semiprime rings, Comm. Algebra 23 (1995), no. 10, 3705-3713.

[5] B. Felzenszwalb, On a result of Levitzki, Canad. Math. Bull. 21 (1978), no. 2, 241-242.

[6] N. Jacobson, Lie Algebras, Dover Publications, New York, 1979.

[7] V. K. Kharchenko, Differential identities of prime rings, Algebra Logic 17 (1978), 155-168.

[8] C. Lanski, Differential identities, Lie ideals, and Posner's theorems, Pacific J. Math. 134 (1988), no. 2, 275-297.

[9] _ An Engel condition with derivation, Proc. Amer. Math. Soc. 118 (1993), no. 3, 731734.

[10] T. K. Lee, Semiprime rings with differential identities, Bull. Inst. Math. Acad. Sinica 20 (1992), no. 1, 27-38.

[11] W. S. Martindale, III, Prime rings satisfying a generalized polynomial identity, J. Algebra 12 (1969), 576-584. 
[12] E. C. Posner, Derivations in prime rings, Proc. Amer. Math. Soc. 8 (1957), 1093-1100.

[13] L. H. Rowen, Polynomial Identities in Ring Theory, Pure and Applied Mathematics, vol. 84, Academic Press, New York, 1980.

[14] J. Vukman, Commuting and centralizing mappings in prime rings, Proc. Amer. Math. Soc. 109 (1990), no. 1, 47-52.

Vincenzo De Filippis: Dipartimento di Matematica, Universitá di Messina, Contrada Papardo, Salita Sperone 31, 98166 Messina, Italy

E-mail address: enzo@dipmat.unime.it 


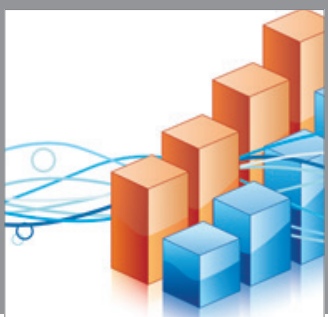

Advances in

Operations Research

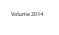

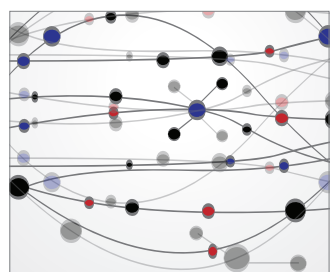

\section{The Scientific} World Journal
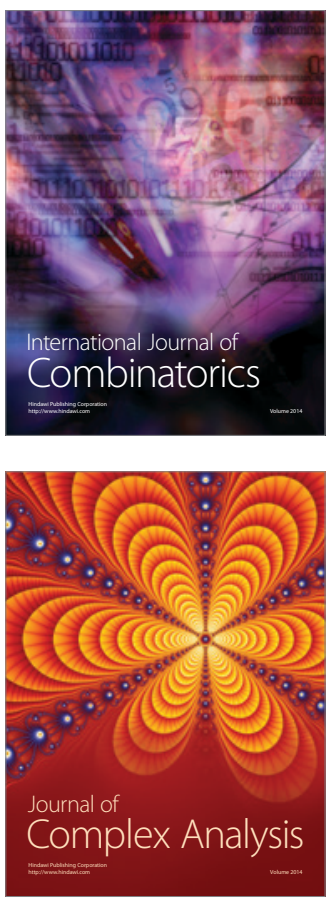

International Journal of

Mathematics and

Mathematical

Sciences
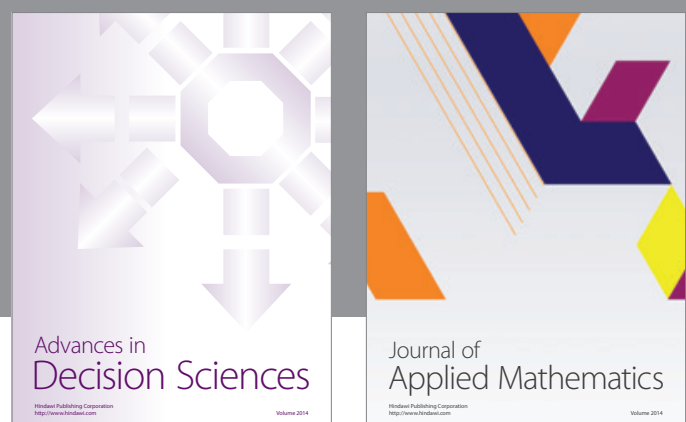

Journal of

Applied Mathematics
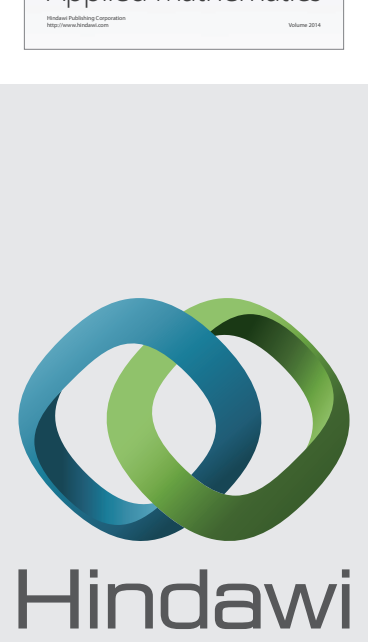

Submit your manuscripts at http://www.hindawi.com
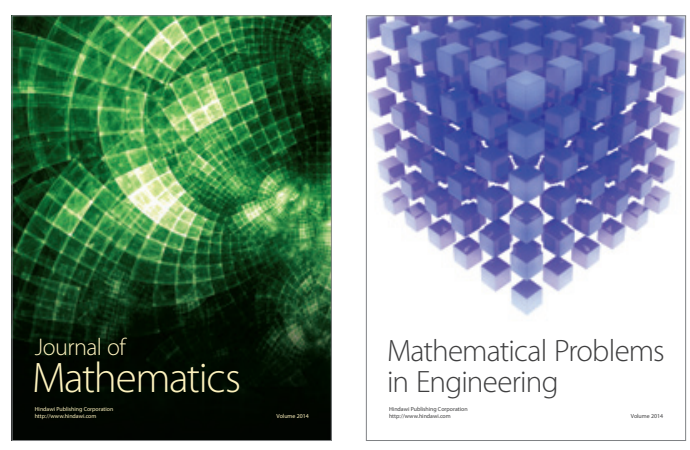

Mathematical Problems in Engineering
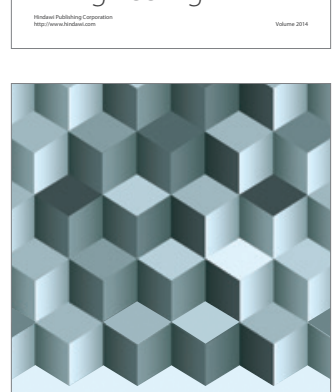

Journal of

Function Spaces
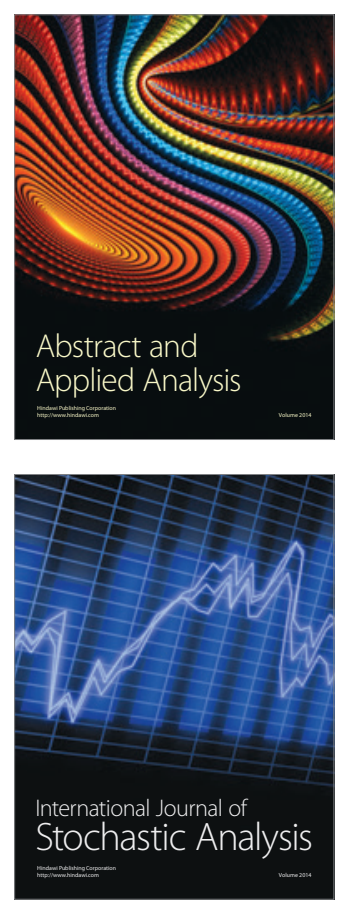

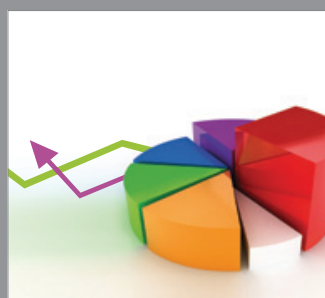

ournal of

Probability and Statistics

Promensencen
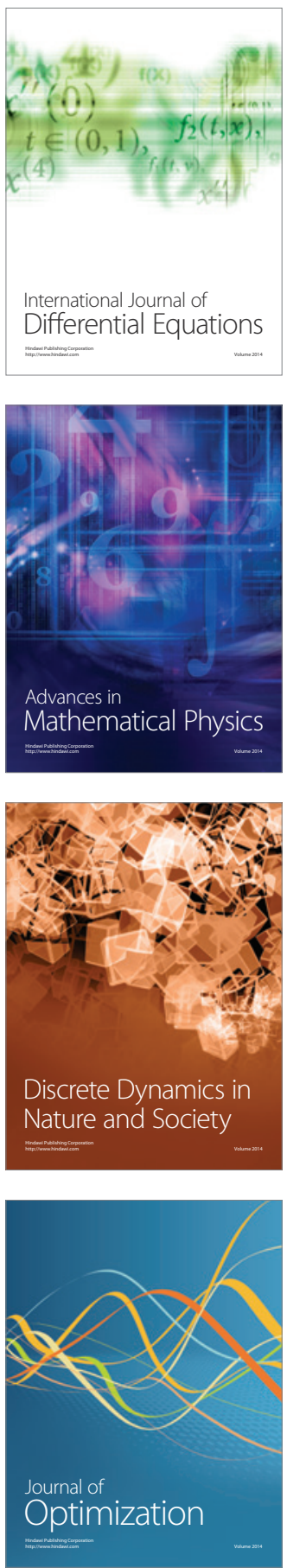